\title{
Pacific
}

Journal of

Mathematics

\section{UNITAL QUADRATIC QUASI-JORDAN ALGEBRAS}

RAÚL FELIPE

Volume $251 \quad$ No. 1

May 2011 


\title{
UNITAL QUADRATIC QUASI-JORDAN ALGEBRAS
}

\author{
RAÚl FELIPE
}

\begin{abstract}
Forty-six years ago, McCrimmon defined the notion of a unital quadratic Jordan algebra. Here we introduce and study the notion of a unital quadratic quasi-Jordan algebra, following earlier work by Loday, Velasquez and the author.
\end{abstract}

\section{Introduction}

In the past century, among nonassociative systems, Jordan algebras and unital quadratic Jordan algebras have occupied a very special place. For instance, Jordan algebras occur in quantum mechanics in connection with the representation of physical observables from an algebraic point of view.

It is well known that an associative algebra $A$ gives rise to a Jordan algebra $A^{+}$ via the Jordan product $x \circ y=\frac{1}{2}(x y+y x)$; it also gives rise to a Lie algebra by means of the product $[x, y]=x y-y x$. A Jordan algebra is called special if it is isomorphic to a subalgebra of a Jordan algebra $A^{+}$for some associative algebra $A$; otherwise it is exceptional. A major problem in the theory of Jordan algebra has been, from the beginning, the classification of simple Jordan algebras. Its solution began with the works of Jordan, von Neumann, Wigner and Albert around 1934 for finite-dimensional algebras and was concluded with Zelmanov's outstanding work in the general case [Albert 1934; Jordan et al. 1934; Zelmanov 1979; 1983].

Jordan algebras also play an important role in others areas of mathematics, such as differential geometry (exceptional algebras; see for instance [Bertram 2000]), and the analysis of nonconvex optimization problems over symmetric cones (specifically, Euclidean Jordan algebras; see [Faybusovich 1997] for more details).

Unital quadratic Jordan algebras were introduced by McCrimmon [1966; 1978] in order to understanding Jordan structures where there is no scalar $\frac{1}{2}$, which necessitate a quadratic approach based in the product $x y x$ instead of $x \circ y=\frac{1}{2}(x y+y x)$. McCrimmon developed this concept to introduce uniform methods in the study

This research was supported in part under CONACYT grant no. 106923 and in part under the Cuba National Research Project Theory and efficient algorithms for the solution of problems in operator algebra and geometric modeling.

MSC2000: primary 17C50; secondary 16W10.

Keywords: Dialgebras, Leibniz algebras, quasi-Jordan algebras. 
of Jordan algebras over characteristic 2. In a strict sense, unital quadratic Jordan algebras are not algebras, because they do not have a bilinear product; however, their connection to Jordan algebras motivated this terminology.

More recently, Loday [1993; 2001] discovered interesting generalizations of associative and Lie algebras, which are now well known as dialgebras and Leibniz algebras. All this leads in a natural way to the question of finding a similar analogue for Jordan algebras, and study the unital quadratic Jordan algebras associated to these new structures. With this purpose, we introduced in [Velásquez and Felipe 2008] the notion of quasi-Jordan algebras.

More specifically, a Leibniz algebra is a generalization of a Lie algebra where the skew-symmetry of the bracket is dropped and the Jacobi identity is changed by the Leibniz identity. Loday observed that the relationship between Lie algebras and associative algebras translate into an analogous relationship between Leibniz algebras and so-called dialgebras, which are a generalization of associative algebras possessing two products: Namely, a dialgebra over a field $K$ is a $K$-vector space $D$ equipped with two associative products

$$
\dashv: D \times D \rightarrow D, \quad \vdash: D \times D \rightarrow D
$$

satisfying the identities

$$
\begin{aligned}
& x \dashv(y \dashv z)=x \dashv(y \vdash z), \\
& (x \vdash y) \dashv z=x \vdash(y \dashv z), \\
& (x \vdash y) \vdash z=(x \dashv y) \vdash z .
\end{aligned}
$$

We say that $e \in D$ is a bar unit of $D$ if for all $x \in D$ we have $e \vdash x=x=x \dashv e$.

Loday showed that any dialgebra $(D, \vdash, \dashv)$ becomes a Leibniz algebra under the Leibniz bracket $[x, y]=x \dashv y-y \vdash x$.

Our notion of quasi-Jordan algebra bears to Leibniz algebras a relationship similar to the one between Jordan algebras and Lie algebras. More precisely, in [Velásquez and Felipe 2008] we attached a quasi-Jordan algebra $Q J_{x}$ to any QJordan element $x$ in a Leibniz algebra. Soon, Kolesnikov [2008] and Bremner [2010] (see also [Bremner and Peresi 2010]) found independently an interesting particular case of quasi-Jordan algebras, in which the analysis of its derivations has a promising future (see [Felipe 2009]). We observe that in a dialgebra over a field of characteristic other than 2 the Jordan quasiproduct takes the form

$$
x \triangleleft y:=\frac{1}{2}(x \dashv y+y \vdash x) .
$$

In other words, any dialgebra over a field of characteristic other than 2 leads to a quasi-Jordan algebra. 
In this paper we generalize the notion of unital quadratic Jordan algebras, beginning with dialgebras. As we will see, one arrives to a new structure (the unital quadratic quasi-Jordan algebra) which include the notion introduced by McCrimmon in 1966.

\section{Definitions and basic examples}

Definition 1 [McCrimmon 2004, page 83]. A unital quadratic Jordan algebra $J$ consists of a $\Phi$-module on which a product $U_{x} y$ is defined which is linear in $y$ and quadratic in $x$ (i.e., $U: x \mapsto U_{x}$ is a mapping of $J$ into $\operatorname{End}_{\Phi}(J)$, homogeneous of degree 2), together with a choice of a unit element $e$, such that the following operator identities hold, where we have defined

$$
V_{x, y} z=\left(U_{x+z}-U_{x}-U_{z}\right) y
$$

for all $x, y, z \in J$ :

(a) $U_{e}=\mathrm{Id}$.

(b) $V_{x, y} U_{x}=U_{x} V_{y, x}$.

(c) $U_{U_{x} y}=U_{x} U_{y} U_{x}$.

Any associative algebra $A$ determines a quadratic Jordan algebra $Q A^{+}$with the product $U_{x} y=x y x$.

In his original paper, McCrimmon [1966] included in the definition of unital quadratic Jordan algebras the condition that the identities (b) and (c) remain valid under extensions of the ring of scalars, and pointed out that this condition is equivalent to the assumption that the linearizations of the identities hold. He subsequently eliminated this requirement [1978; 2004]. We return to this point in Section 3.

Definition 2. A unital quadratic quasi-Jordan algebra over a field $K$ is a quadruple $(\mathfrak{I}, U, W, e)$, where $\mathfrak{I}$ is a $K$-vector space, $e$ is a distinguished element of $\mathfrak{s}$, and $U$ and $W$ are maps $a \mapsto U_{a}$ and $a \mapsto W_{a}$ of $\Im$ into $\operatorname{End}_{K}(\Im)$ satisfying the following axioms:

(QQJ1) $U_{e}=\operatorname{Id}$ and $W_{e} e=e$.

(QQJ2) $W_{z} U_{x} V_{y, x}=W_{z} V_{x, y} U_{x}$ for all $x, y, z \subset \mathfrak{s}$, in the notation of (5).

(QQJ3) $U_{U_{x} y}=U_{x} U_{y} U_{x}$, for every $x, y \subset \Im$.

(QQJ4) $U_{\lambda x} e=\lambda^{2} U_{x} e$ for any $x \in \mathfrak{I}$.

We say that $e$ is the unit of the unital quadratic quasi-Jordan algebra.

The need for a second operator $W$ arises as follows. We wish to include split quasi-Jordan algebras (where the product $\triangleleft$ is right commutative) among unital quadratic quasi-Jordan algebras. But in general, it is not true that $U_{x} V_{y, x}=V_{x, y} U_{x}$ 
for unital quadratic quasi-Jordan algebras, as will become clear after Lemma 4 . The operator $W$ is responsible, so to speak, for ensuring that $U_{(\cdot)}$ and $V_{(\cdot, \cdot)}$ "commute" (QQJ2). Moreover, we want to be able to construct quasi-Jordan algebras from unital quadratic quasi-Jordan algebras (Section 4).

Lemma 3. Any unital quadratic Jordan algebra is a unital quadratic quasi-Jordan algebra in which $W_{a}=U_{a}$ for all $a \in \mathfrak{I}$. In this case $U_{x}$ is $K$-quadratic with respect to $x$.

Proof. This is immediately checked from the definitions.

The real motivation for Definition 2 is the following lemma.

Lemma 4. Let $(D, \vdash, \dashv, e)$ be a unital $K$-dialgebra. We need not suppose that the field $K$ is of characteristic other than 2. Define

$$
U_{x} y=(x \vdash y) \dashv x=x \vdash(y \dashv x), \quad W_{x} y=(x \dashv y) \dashv x=x \dashv(y \dashv x) .
$$

Then $(D, U, W, e)$ is a unital quadratic quasi-Jordan algebra, for which $U$ and $W$ are homogeneous of degree 2 (as maps $D \rightarrow \operatorname{End}_{K}(D)$ ).

The unital quadratic quasi-Jordan algebra built from a unital dialgebra $D$ will be denoted by $(Q Q(D), e)$.

Proof. It is clear that $U_{e} x=x$ for all $x \in D$. Next, $W_{e} e=(e \dashv e) \dashv e=e$. The homogeneity condition - that is, $U_{\lambda x} y=\lambda^{2} U_{x} y$ and $W_{\lambda x} y=\lambda^{2} W_{x} y$ for any $x, y \in \mathfrak{I}$ and any scalar $\lambda$ - is also easy to check.

To show that QQJ3 holds, we write

$$
\begin{aligned}
U_{U_{x}} z & =U_{(x \vdash y) \dashv x} z=(((x \vdash y) \dashv x) \vdash z) \dashv((x \vdash y) \dashv x) \\
& =((x \vdash y) \dashv x) \vdash(z \dashv((x \vdash y) \dashv x)) \\
& =((x \vdash y) \dashv x) \vdash(z \dashv(x \vdash(y \dashv x))) \\
& =((x \vdash y) \dashv x) \vdash(z \dashv(x \dashv(y \dashv x))) \\
& =((x \vdash y) \vdash x) \vdash(z \dashv(x \dashv(y \dashv x))) \\
& =(x \vdash y) \vdash(x \vdash(z \dashv(x \dashv(y \dashv x)))) \\
& =(x \vdash y) \vdash\left(\left(U_{x} z\right) \dashv(y \dashv x)\right) \\
& =U_{x} U_{y} U_{x} z .
\end{aligned}
$$

To simplify the rest of the proof we introduce some notation. If $a_{1}, a_{2}, \ldots, a_{n}$ are elements of $D$ and $1 \leq k \leq n$, we set

$$
\begin{aligned}
& a_{1} a_{2} \ldots a_{k-1} \widehat{a_{k}} a_{k+1} \ldots a_{n-1} a_{n} \\
& \quad=\left(a_{1} \vdash a_{2} \vdash \ldots \vdash a_{k-2} \vdash a_{k-1}\right) \vdash a_{k} \dashv\left(a_{k+1} \dashv a_{k+2} \dashv \ldots \dashv a_{n-1} \dashv a_{n}\right),
\end{aligned}
$$

where the right-hand side is well defined by associativity. 
Next we verify the axiom QQJ2. We have

$$
\begin{aligned}
W_{c} U_{x} V_{y, x} z & =W_{c} U_{x}[(y \vdash x) \dashv z+(z \vdash x) \dashv y] \\
& =W_{c}[(x \vdash((y \vdash x) \dashv z)) \dashv x+(x \vdash((z \vdash x) \dashv y)) \dashv x] \\
& =(c \dashv((x \vdash((y \vdash x) \dashv z)) \dashv x)) \dashv c+(c \dashv((x \vdash((z \vdash x) \dashv y)) \dashv x)) \dashv c \\
& =\widehat{c} x y x z x c+\widehat{c} x z x y x c ;
\end{aligned}
$$

on the other hand

$$
\begin{aligned}
W_{c} V_{x, y} U_{x} z & =W_{c} V_{x, y}((x \vdash z) \dashv x) \\
& =W_{c}[((x \vdash y) \dashv((x \vdash z) \dashv x))+((((x \vdash z) \dashv x) \vdash y) \dashv x)] \\
& =(c \dashv((x \vdash y) \dashv((x \vdash z) \dashv x))) \dashv c+(c \dashv((((x \vdash z) \dashv x) \vdash y) \dashv x)) \dashv c \\
& =\widehat{c} x y x z x c+\widehat{c} x z x y x c .
\end{aligned}
$$

Thus, QQJ2 follows. Finally that $U_{x}$ and $W_{x}$ belong to $\operatorname{End}_{K}(D)$ for any $x \in D$ is evident.

It is not hard to see that $U_{x} V_{y, x}$ and $V_{x, y} U_{x}$ need not coincide for unital quadratic quasi-Jordan algebras. In fact, from the proof of Lemma 4 it follows that

$$
\begin{aligned}
& U_{x} V_{y, x} z=(x \vdash((y \vdash x) \dashv z)) \dashv x+(x \vdash((z \vdash x) \dashv y)) \dashv x, \\
& V_{x, y} U_{x} z=((x \vdash y) \dashv((x \vdash z) \dashv x))+((((x \vdash z) \dashv x) \vdash y) \dashv x) .
\end{aligned}
$$

Taking $x=e$, one obtains from (6) that $U_{e} V_{y, e} z=y \vdash e \dashv z+z \vdash e \dashv y$, and from (7) that $V_{e, y} U_{e} z=y \dashv z+z \vdash y$. Thus, for nonzero $y \in Z_{B}(D)$ we have $U_{e} V_{y, e} e=0$, but $V_{e, y} U_{e} e=2 y$, which is nonzero if the characteristic is not 2 .

\section{Linearization}

We now turn to the "linearization interpretation" of the axioms in Definition 2. We restrict ourselves to the case of unital quadratic quasi-Jordan algebras $(Q Q(D), e)$.

Recall that in the proof of Lemma 4 we used the equality

$$
V_{x, y} z=(x \vdash y) \dashv z+(z \vdash y) \dashv x .
$$

Recall also that $U_{x} y=(x \vdash y) \dashv x$. If we replace $x$ by $x+\alpha z$ in this latter equality, we obtain

$$
U_{x+\alpha z} y=U_{x} y+\left(V_{x, y} z\right) \alpha+\left(U_{z} y\right) \alpha^{2} ;
$$

that is, we can consider to $V_{x, y}$ as the "linearization" of $U_{x}$, which justifies its presence in axiom QQJ2.

One can see, after a cumbersome calculation, that if the field of scalars over which a unital quadratic quasi-Jordan algebra $(Q Q(D), e)$ is defined has at least 
four elements, the linearization of QQJ2 is

(8) $W_{v}\left(U_{x} V_{y, w} z+V_{w, V_{y, x} z} x\right)=W_{v}\left(V_{w, y} U_{x} z+V_{x, y} V_{w, z} x\right) \quad$ for $v, x, y, z, w \in D$.

If the field of scalars has at least five elements, linearizing QQJ3 we obtain

$$
U_{x} U_{y} V_{x, w} z+V_{w, U_{y} U_{x} z} x=V_{V_{w, y} x, z} U_{x} y,
$$

for all $x, y, z, w \in D$. Thus, if $D$ is a dialgebra with a bar unit defined over a field with at least five elements, the axioms QQJ2 and QQJ3 for $(Q Q(D), e)$ can be linearized in the form (8) and (9) respectively.

\section{Relation to quasi-Jordan algebras}

Let $(D, \vdash, \dashv, e)$ be a unital dialgebra. The unital quadratic quasi-Jordan algebra $(Q Q(D), e)$ is restrictive, that is, it satisfies the condition

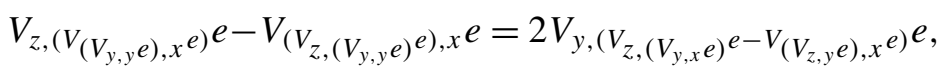

for all $x, y, z \in \mathfrak{\Im}$. Indeed, (10) is the Bremner-Kolesnikov identity for the quasiJordan product defined from dialgebras [Bremner 2010; Felipe 2009; Kolesnikov 2008].

It is well known that any unital Jordan algebra $(J, \bullet, e)$ over a field of characteristic other than 2 gives rise to a unital quadratic Jordan algebra (and so also a unital quadratic quasi-Jordan algebra) for which, if $R_{x} y$ denotes the product of $y$ by $x$,

$$
U_{x} y=\left(2 R_{x}^{2}-R_{x^{2}}\right) y \quad \text { and } \quad x \cdot y=\frac{1}{2}\left(U_{x+y}-U_{x}-U_{y}\right) e=K_{x, y} e .
$$

At the same time, Bremner [2010] has shown that the Bremner-Kolesnikov identity holds in Jordan algebras. Hence, we have

$$
K_{\left(K_{a,\left(K_{b, b} e\right)} e\right), c} e-K_{a,\left(K_{\left(K_{b, b}\right), c} e\right)} e=2 K_{\left(K_{\left(K_{a, b} e\right),} e-K_{a,\left(K_{b, c}\right.} e\right)^{e), b}} e,
$$

for all $a, b, c \in J$. Since $V_{x, y}$ and $K_{x, y}$ act differently on a element, this last equality is distinct from (10). This is not surprising, because in general the quasi-Jordan algebra arising from a dialgebra is not a Jordan algebra.

We know that by means of the right and left products of a $K$-dialgebra over a field $K$ of characteristic other than 2, we can build a new product on the same underlying vector space (see below after the next definition) with respect to which it becomes a quasi-Jordan algebra (in fact, this new product is right commutative). See [Velásquez and Felipe 2008; 2009] for details.

Definition 5. A quasi-Jordan algebra is a vector space $\mathfrak{I}$ over a field $K$ of a characteristic other than 2 equipped with a bilinear product $\triangleleft: \mathfrak{s} \times \mathfrak{s} \rightarrow \mathfrak{s}$ such that

$$
x \triangleleft(y \triangleleft z)=x \triangleleft(z \triangleleft y) \quad \text { (right commutativity) }
$$


and

$$
(y \triangleleft x) \triangleleft x^{2}=\left(y \triangleleft x^{2}\right) \triangleleft x \quad \text { (right Jordan identity) }
$$

for all $x, y, z \in \mathfrak{I}$, where $x^{2}=x \triangleleft x$. A unit of a quasi-Jordan algebra $\mathfrak{I}$ is an element $e \in \mathfrak{I}$ such that $x \triangleleft e=x$ for all $x \in \mathfrak{I}$.

Example 6. As noted earlier, quasi-Jordan algebras appear in the study of the product

$$
x \triangleleft y:=\frac{1}{2}(x \dashv y+y \vdash x),
$$

where $x$ and $y$ are elements in a dialgebra $(D, \vdash, \dashv)$ over a field $K$ of characteristic other than 2. The quasi-Jordan algebra defined over $D$ with the product (13) is denoted by $(\Im(D), \triangleleft)$.

From the results above we see that if $D$ has a bar unit $e$, our construction defines over $D$ a unital quadratic quasi-Jordan algebra $(Q Q(D), e)$. In this case we have: Lemma 7. For any $x \in Q Q(D)$, the linear transformation $U_{x}$ can be recovered as

$$
U_{x} y=\left(2 R_{x}^{2}-R_{x^{2}}\right) y,
$$

where $R_{x}$ is right multiplication by $x$ (that is, the element of $\operatorname{End}(\Im(D))$ defined by $\left.R_{x} y=y \triangleleft x\right)$. The product $\triangleleft$ in $((\Im(D), \triangleleft), e)$ is recovered as $y \triangleleft x=\frac{1}{2} V_{x, y}$.

Proof. We prove the first statement; the proof of the equality $y \triangleleft x=\frac{1}{2} V_{x, y} e$ is similar. In fact,

$$
\begin{aligned}
& \left(2 R_{x}^{2}-R_{x^{2}}\right) y \\
& \quad=2(y \triangleleft x) \triangleleft x-y \triangleleft(x \triangleleft x) \\
& =\frac{1}{2}((y \dashv x+x \vdash y) \dashv x+x \vdash(y \dashv x+x \vdash y))-\frac{1}{4}(y \dashv(x \dashv x+x \vdash x)+(x \dashv x+x \vdash x) \vdash y) \\
& =(x \vdash y) \dashv x=U_{x} y .
\end{aligned}
$$

For a quasi-Jordan algebra $\Im$ we introduce

$$
Z^{r}(\mathfrak{I})=\{z \in \mathfrak{I}: x \triangleleft z=0 \text { for all } x \in \mathfrak{I}\} .
$$

We denote by $\Im^{\text {ann }}$ the subspace of $\Im$ spanned by elements of the form $x \triangleleft y-y \triangleleft x$, with $x, y \in \mathfrak{I}$, and call it the annihilator ideal of the quasi-Jordan algebra $\mathfrak{I}$. Then $\Im$ is a Jordan algebra if and only if $\Im^{\text {ann }}=\{0\}$. It follows from right commutativity (11) that in any quasi-Jordan algebra

$$
x \triangleleft(y \triangleleft z-z \triangleleft y)=0 .
$$

The last identity implies that $\Im^{\text {ann }} \subset Z^{r}(\Im)$. One can prove that both $\Im^{\text {ann }}$ and $Z^{r}(\Im)$ are two-sided ideals of $\Im$. Now recall from [Velásquez and Felipe 2008] 
that if $\mathfrak{s}$ is a unital quasi-Jordan algebra, with a specific unit $e$, then

$$
\mathfrak{\Im}^{\mathrm{ann}}=Z^{r}(\Im), \quad \Im^{\mathrm{ann}}=\{x \in \Im: e \triangleleft x=0\} .
$$

It is now clear that units in quasi-Jordan algebras are not unique; indeed, the set of units $U_{r}(\mathfrak{\Im})$ of $\Im$ is given by

$$
U_{r}(\mathfrak{I})=\left\{x+e: x \in \mathfrak{\Im}^{\mathrm{ann}}\right\} .
$$

Definition 8. Let $\Im$ be a quasi-Jordan algebra and let $I$ be an ideal in $\Im$ such that $\mathfrak{\Im}^{\text {ann }} \subset I \subset Z^{r}(\Im)$. We say that $\Im$ is split over $I$ if there is a subalgebra $J$ of $\Im$ such that $\mathfrak{s}=I \oplus J$ as a direct sum of subspaces.

Clearly, if $\mathfrak{I}$ is split over an ideal $I$ with complement $J$, then $J$ is a Jordan algebra with respect to the product $\triangleleft$ restricted to $J$. This is equivalent to saying that $\left(J,\left.\triangleleft\right|_{J}\right)$ is a Jordan algebra. In fact, for $x, y \in J$, then $x \triangleleft y, y \triangleleft x \in J$ and $x \triangleleft y-y \triangleleft x \in I \cap J=\{0\}$; that is, $\left.\triangleleft\right|_{J}$ is commutative and therefore the right Jordan identity over $\Im$ implies that $\left(J,\left.\triangleleft\right|_{J}\right)$ is a Jordan algebra.

Additionally, for $a, b \in I$ and $x, y \in J$ we have

$$
(a+x) \triangleleft(b+y)=a \triangleleft y+x \triangleleft y,
$$

because $I \subset Z^{r}(\Im)$.

Reciprocally, let $(J, \bullet)$ be a Jordan algebra and let $M$ be a Jordan bimodule over $J$. We consider the direct sum $\Im:=M \oplus J$ and we define the product $\triangleleft$ over $\Im$ by

$$
(a+x) \triangleleft(b+y)=a y+x \bullet y,
$$

for all $a, b \in M$ and $x, y \in J$. Then $(\mathfrak{I}, \triangleleft)$ is a quasi-Jordan algebra, called the demisemidirect product of $M$ with $J$.

It is possible to see that $\Im^{\text {ann }} \cong M J$ and

$$
Z^{r}(\mathfrak{I})=M \oplus\{y \in Z(J): u y=0 \text { for all } u \in M\},
$$

where $Z(J)=\{y \in J: x \bullet y=0$ for all $x \in J\}$. Finally, $M \cong M \oplus\{0\}$ is an ideal of $\mathfrak{I}$ such that $\mathfrak{J}^{\text {ann }} \subset M \subset Z^{r}(\mathfrak{I})$. In addition, $\mathfrak{I} / M \cong J$ and $\mathfrak{I}$ is split over $M$ with complement $J$.

Let $(\Im, \bullet)$ be an algebra. Assume that $\mathfrak{\Im}=I \oplus J$, where $(J, \bullet)$ is a Jordan algebra and $I$ is an ideal of $\mathfrak{I}$. In general $I$ is not a Jordan bimodule over $J$ with respect to the product $\bullet$. However, we can define a new product on $\mathfrak{s}$ by

$$
(a+x) \triangleleft(b+y)=a \bullet y+x \bullet y,
$$

for all $a, b \in I$ and $x, y \in J$.

Lemma 9. Let $(\Im, \bullet)$ be an algebra such that $\Im=I \oplus J$, where $(J, \bullet)$ is a Jordan algebra and $I$ is an ideal of $\Im$. Suppose that $\left(a \bullet x^{2}\right) \bullet x=(a \bullet x) \bullet x^{2}$ for all $a \in I$ 
and $x \in J$, where $x^{2}=x \bullet x$. Then $(\Im, \triangleleft)$ is a quasi-Jordan algebra, where $\triangleleft$ is the product defined by (15). Moreover $\Im^{\text {ann }} \subset I \subset Z^{r}(\mathfrak{I})$.

We refer to $(\Im, \triangleleft)$ as the demisemidirect product of $I$ with $J$.

Proof. The product (15) is right commutative; in fact, if $a, b, c \in I$ and $x, y, z \in J$,

$$
\begin{aligned}
(a+x) \triangleleft((b+y) \triangleleft(c+z)) & =a \bullet(y \bullet z)+x \bullet(y \bullet z) \\
& =a \bullet(z \bullet y)+x \bullet(z \bullet y) \\
& =(a+x) \triangleleft((c+z) \triangleleft(b+y)) .
\end{aligned}
$$

Observe that $(a+x) \triangleleft(a+x)=a \cdot x+x^{2}$. Now

$$
\begin{aligned}
((b+y) \triangleleft(a+x)) \triangleleft\left(a \bullet x+x^{2}\right) & =(b \bullet x) \bullet x^{2}+(y \bullet x) \bullet x^{2} \\
& =\left(b \bullet x^{2}\right) \bullet x+\left(y \bullet x^{2}\right) \bullet x \\
& =\left((b+y) \triangleleft\left(a \cdot x+x^{2}\right)\right) \triangleleft(a+x) .
\end{aligned}
$$

Thus, the right Jordan identity holds. On the other hand,

$$
(a+x) \triangleleft(b+y)-(b+y) \triangleleft(a+x)=a \bullet y-b \bullet x \in I .
$$

It shows that $\mathfrak{\Im}^{\text {ann }} \subset I$. Finally, we have

$$
(a+x) \triangleleft b=(a+x) \triangleleft(b+0)=a \bullet 0+x \bullet 0=0,
$$

which implies that $I \subset Z^{r}(\Im)$.

Theorem 10. Let $\Im$ be a quasi-Jordan algebra and let I be an ideal of $\Im$ such that $\mathfrak{\Im}^{\text {ann }} \subset I \subset Z^{r}(\Im)$. Then $\Im$ is split over $I$ if and only if $\Im$ is the demisemidirect product of I with a Jordan algebra J.

Proof. This follows from Lemma 9 and the discussion preceding that lemma.

The property of being a split quasi-Jordan algebra is important for us, among other reasons because every quasi-Jordan algebra is isomorphic to a subalgebra of a split quasi-Jordan algebra.

Now suppose that $\Im$ is a split quasi-Jordan algebra with a specific unit $e$. Since, by (14), $\Im^{\text {ann }}$ and $Z^{r}(\Im)$ coincide, there is a Jordan algebra $J$ such that $\Im=\Im^{\text {ann }} \oplus J$.

Because $e \in \mathfrak{I}$ is a unit in $\mathfrak{I}$, there are elements $a \in \mathfrak{I}^{\text {ann }}$ and $\epsilon \in J$ such that $e=a+\epsilon$. If $b+y \in \mathfrak{I}$, with $b \in \mathfrak{I}^{\text {ann }}$ and $y \in J$, we have

$$
b+y=(b+y) \triangleleft e=(b+y) \triangleleft(a+\epsilon)=b \triangleleft \epsilon+y \triangleleft \epsilon=(b+y) \triangleleft \epsilon .
$$

The last equality implies that $\epsilon$ is a unit in $\Im$ and a unit in the Jordan algebra $J$. Also, $\epsilon$ is the only element in $J$ such that $a+\epsilon$ is a unit in $\Im$ for all $a \in \Im^{\text {ann }}$. This shows that the units in a split quasi-Jordan algebra are of the form $a+\epsilon$, where $a \epsilon$ $\mathfrak{\Im}^{\text {ann }}$ and $\epsilon$ is the unique unit of a unital Jordan algebra; hence $U_{r}(\mathfrak{I})=\Im^{\text {ann }} \oplus\{\epsilon\}$. 
Theorem 11. Let $\Im=\Im^{\text {ann }} \oplus J$ be a unital split quasi-Jordan algebra and $\epsilon \in J$ a unit of $\Im$ which is also the unique unit of the Jordan algebra J. Then $(\Im, U, W, \epsilon)$ is a unital quadratic quasi-Jordan algebra in which $U$ and $W$ are defined as follows (if $x, y \in J$, we denote the product of $x$ with $y$ by $x y$ instead of $x \triangleleft y$ ):

$$
U_{a+x}(b+y)=b+U_{x} y, \quad W_{a+x}(b+y)=-a \triangleleft y+(x y),
$$

where $a, b \in \mathfrak{\Im}^{\mathrm{ann}}, x, y \in J$ and $U_{x} y=\left(2 R_{x}^{2}-R_{x^{2}}\right) y$. Here $R_{x} y=y x=x y$.

As the reader probably has noticed, where no misunderstanding can arise, we will use the letter $U$ to denote simultaneously the map $U_{a+x}$ for any $a+x \in \mathfrak{I}$ and the map $U_{z}$ for every $z \in J$.

Proof. Keep in mind that $J$ is a Jordan algebra. We have $U_{\epsilon}(b+y)=b+U_{\epsilon} y=$ $b+y$; thus $U_{\epsilon}=I_{d}$. At the same time, $W_{\epsilon} \epsilon=\epsilon$.

Obviously $U_{a+x}(b+y)$ and $W_{a+x}(b+y)$ are linear with respect to $(b+y)$ and $U_{\lambda(a+x)} \epsilon=U_{\lambda x} \epsilon=\lambda^{2} U_{x} \epsilon=\lambda^{2} U_{a+x} \epsilon$.

Next,

$$
U_{U_{a+x}(b+y)}(c+z)=U_{b+U_{x} y}(c+z)=c+U_{U_{x} y} z .
$$

On the other hand,

$$
\begin{aligned}
U_{a+x} U_{b+y} U_{a+x}(c+z) & =U_{a+x} U_{b+y}\left(c+U_{x} z\right) \\
& =U_{a+x}\left(c+U_{y} U_{x} z\right)=c+U_{x} U_{y} U_{x} z ;
\end{aligned}
$$

since $U_{U_{x} y} z=U_{x} U_{y} U_{x} z$. From (17) and (18) we have

$$
U_{U_{a+x}(b+y)}=U_{a+x} U_{b+y} U_{a+x} .
$$

Next we check condition QQJ2. First we obtain

$$
\begin{aligned}
V_{(b+y),(a+x)}(c+z) & =\left(U_{((b+c)+(y+z))}-U_{(b+y)}-U_{(c+z)}\right)(a+x) \\
& =\left(a+U_{y+z} x\right)-\left(a+U_{y} x\right)-\left(a+U_{z} x\right) \\
& =-a+\left(U_{y+z} x-U_{y} x-U_{z} x\right)=-a+V_{y, x} z .
\end{aligned}
$$

Similarly, $V_{(a+x),(b+y)}(c+z)=-b+V_{x, y} z$. Hence

$$
U_{a+x} V_{(b+y),(a+x)}(c+z)=U_{a+x}\left(-a+V_{y, x} z\right)=-a+U_{x} V_{y, x} z,
$$

which implies that

$$
\begin{aligned}
W_{d+w} U_{a+x} V_{(b+y),(a+x)}(c+z) & =W_{d+w}\left(-a+U_{x} V_{y, x} z\right) \\
& =-d \triangleleft\left(U_{x} V_{y, x} z\right)+w\left(U_{x} V_{y, x} z\right) .
\end{aligned}
$$

Observe also that

$$
V_{(a+x),(b+y)} U_{a+x}(c+z)=V_{(a+x),(b+y)}\left(c+U_{x} z\right)=-b+V_{x, y} U_{x} z,
$$


and from this we conclude that

$$
\begin{aligned}
W_{d+w} V_{(a+x),(b+y)} U_{a+x}(c+z) & =W_{d+w}\left(-b+V_{x, y} U_{x} z\right) \\
& =-d \triangleleft\left(V_{x, y} U_{x} z\right)+w\left(V_{x, y} U_{x} z\right) .
\end{aligned}
$$

Using the commutativity property $U_{x} V_{y, x}=V_{x, y} U_{x}$ of Jordan algebras, it follows from (20) and (21) that $W_{d+w} U_{a+x} V_{(b+y),(a+x)}=W_{d+w} V_{(a+x),(b+y)} U_{a+x}$ for all $(a+x),(b+y),(d+w) \in \Im$. This concludes the proof of the theorem.

Let $\mathfrak{I}=\mathfrak{\Im}^{\text {ann }} \oplus J$ be a unital split quasi-Jordan algebra with $\epsilon \in J$ as unit, then we denote $\wp(\Im)$ for the unital quadratic quasi-Jordan algebra $(\Im, U, W, \epsilon)$ corresponding to the previous theorem.

\section{Split unital quadratic quasi-Jordan algebras}

For a unital quadratic quasi-Jordan algebra $(\Im, U, W, e)$ we put

$$
Z^{r}(\mathfrak{S})=\left\{z \in \mathfrak{S}: W_{x} z=0 \text { for all } x \in \mathfrak{I}\right\} .
$$

We denote by $\mathfrak{\Im}^{\text {ann }}$ the subspace of $\Im$ spanned by elements of the form

$$
\left(U_{x} V_{y, x}-V_{x, y} U_{x}\right) z, \quad \text { with } x, y, z \in \mathfrak{I} .
$$

$\Im$ is a unital quadratic Jordan algebra if and only if $\Im^{\text {ann }}=\{0\}$ and $U_{x}$ is $K$-quadratic with respect to all $x \in \mathfrak{\Im}$. From QQJ2 follows that $\mathfrak{\Im}^{\text {ann }} \subset Z^{r}(\mathfrak{I})$.

Proposition 12. If ( $\Im, U, W, e$ ) is a unital quadratic quasi-Jordan algebra, the unit e does not belong to $\mathfrak{S}^{\mathrm{ann}}$.

Proof. Otherwise, one can write $e=\sum\left(U_{x_{i}} V_{y_{i}, x_{i}}-V_{x_{i}, y_{i}} U_{x_{i}}\right) z_{i}$, where the sum is finite. Applying $W_{e}$ to this equality and taking into account QQJ1 and QQJ2 we obtain $e=0$, which is impossible.

In fact a more general statement holds: $e$ does not belong to $Z^{r}(\Im)$.

Definition 13. We say that a unital quadratic quasi-Jordan algebra $(\Im, U, W, e)$ is split if there exists a subspace $Q J$ such that $\mathfrak{\Im}=\mathfrak{\Im}^{\text {ann }} \oplus Q J$ as a direct sum of subspaces and $U_{x} Q J \subset Q J$ for all $x \in Q J$.

Lemma 14. Let ( $\Im, U, W, e)$ be a split unital quadratic quasi-Jordan algebra such that $\mathfrak{\Im}=\mathfrak{\Im}^{\mathrm{ann}} \oplus Q J$. Then, if $U$ is $K$-quadratic, $Q J$ is a unital quadratic Jordan algebra.

Proof. Take $x, y, z \in Q J$. We have $\left(U_{x} V_{y, x}-V_{x, y} U_{x}\right) z \in \Im^{\mathrm{ann}} \cap Q J$; therefore

$$
\left(U_{x} V_{y, x}-V_{x, y} U_{x}\right) z=0,
$$

so $U_{x} V_{y, x}=V_{x, y} U_{x}$ for all $x, y \in Q J$. This shows that $\left(Q J, U_{\mid Q J}, W_{\mid Q J}, e\right)$ is a unital quadratic Jordan algebra. 
Now suppose that $(D, \vdash, \dashv, e)$ is a unital split dialgebra such that $D=D^{\text {ann }} \oplus A$, where $A$ is an associative algebra (so $\vdash=\dashv$ on $A$ ) and $e$ is a bar unit of $D$ which is the unique unit of $A$. ( $D^{\text {ann }}$, the annihilator ideal of $D$, is the subspace of $D$ spanned by elements of the form $x \dashv y-x \vdash y$; see [Velásquez and Felipe 2009] for details). Then

$$
(a+i) \dashv(b+j)=(a \dashv j)+i j \quad \text { and } \quad(a+i) \vdash(b+j)=(i \vdash b)+i j,
$$

where $a, b \in D^{\mathrm{ann}}$ and $i, j \in A$, moreover $D^{\mathrm{ann}}$ is spanned by elements of the form $a \dashv i$ and $k \vdash b$.

Theorem 15. If $D=D^{a n n} \oplus A$ is a unital split dialgebra as above, the unital quadratic quasi-Jordan algebra $(Q Q(D), e)$ is split.

Proof. Since $U_{x} y=(x \vdash y) \dashv x=x y x \in A$ if $x, y \in A$, it is sufficient to check that $D^{\text {ann }}=(Q Q(D))^{\text {ann }}$. Now, it is easy to show through calculation that the term in the expression

$$
U_{(a+i)} V_{(b+j),(a+i)}(c+k)-V_{(a+i),(b+j)} U_{(a+i)}(c+k)
$$

that belongs to $A$ is $(i((j i) k)) i+(i((k i) j)) i-((i j)((i k) i))-(((i k) i) j)=T$; but since $A$ is associative we conclude that $T=0$. The remaining four terms are of the form $d \dashv l$ and $m \vdash f$. It follows that $(Q Q(D))^{\text {ann }} \subset D^{\text {ann }}$. On the other hand, taking $i=j=e$ in (22), this expression will be equal to $a \dashv k+k \vdash a-b \dashv k-k \vdash b$. Setting $b=0$ we conclude that the elements of the form $d \dashv l$ and $m \vdash f$ (which span $\left.D^{\text {ann }}\right)$ can be obtained by means of (22). Thus $D^{\text {ann }} \subset(Q Q(D))^{\text {ann }}$. This completes the proof of the theorem.

Proposition 16. Let $\wp(\Im)=(\Im, U, W, \epsilon)$ be the unital quadratic quasi-Jordan algebra associated to a unital split quasi-Jordan algebra $\Im=\Im^{\text {ann }} \oplus J$ with $\epsilon \in J$ as a unit. Then $\wp(\Im)$ is split.

Proof. It follows from (16) that $U_{x} y \in J$ for any $x, y \in J$. At the same time,

$$
\begin{aligned}
\left(U_{a+x} V_{(b+y),(a+x)}-V_{(a+x),(b+y)} U_{a+x}\right)(c+z) & =\left(-a+U_{x} V_{y, x} z\right)-\left(-b+V_{x, y} U_{x} z\right) \\
& =b-a,
\end{aligned}
$$

where $a, b, c \in \Im^{\text {ann }}$ and $x, y, z \in J$. We obtain $\left.b=U_{x} V_{(b+y), x}-V_{x,(b+y)} U_{x}\right)(c+z)$ by setting $a=0$. Since $b \in \Im^{\text {ann }}$ is arbitrary, this implies that $\wp(\Im)^{\text {ann }}=\Im^{\text {ann }}$.

\section{Concluding remarks}

We propose a few possible directions of work:

(i) Inner ideals play a role in the theory of quadratic Jordan algebras analogous to that played by the one-sided ideals in the theory of associative algebras. It 
is therefore important to develop a corresponding ideal theory for quadratic quasi-Jordan algebras.

(ii) Although representations do not play as much of a role in the theory of Jordan algebras as they do in the associative or Lie theories, we propose to develop a representation theory for unital quadratic quasi-Jordan algebras. There exists some previous work of McCrimmon about this subject for quadratic Jordan algebras.

(iii) One of the most controversial concepts about dialgebras and quasi-Jordan algebras, one which is still under study, is that of a regular or invertible element. We think the reason for this is the nonuniqueness of the unit in these algebraic structures. Hence, an interesting subject of study could be the notion of a regular element on a unital quadratic quasi-Jordan algebra. Maybe this could help unify views and opinions in the near future.

(iv) There are some techniques for establishing identities in Jordan algebras and quadratic Jordan algebras, among which the best known are Macdonald's principle, Kocher's principle and McCrimmon's principle. It would be useful to find corresponding principles for unital quadratic quasi-Jordan algebras with the help of which we may know, for instance, whether (8) and (9) hold for any unital quadratic quasi-Jordan algebra.

\section{Acknowledgment}

The author thanks the anonymous referee for his/her careful reading and remarks which helped to improve the manuscript.

\section{References}

[Albert 1934] A. A. Albert, "On a certain algebra of quantum mechanics", Ann. of Math. 35:1 (1934), 65-73. MR 1503142 Zbl 0008.42104

[Bertram 2000] W. Bertram, The geometry of Jordan and Lie structures, Lecture Notes in Math. 1754, Springer, Berlin, 2000. MR 2002e:17041 Zbl 1014.17024

[Bremner 2010] M. R. Bremner, "On the definition of quasi-Jordan algebra”, Comm. Algebra 38:12 (2010), 4695-4704. Zbl 05859516

[Bremner and Peresi 2010] M. R. Bremner and L. A. Peresi, "Special identities for quasi-Jordan algebras", preprint, 2010. arXiv 1008.2723

[Faybusovich 1997] L. Faybusovich, "Euclidean Jordan algebras and interior-point algorithms", Positivity 1:4 (1997), 331-357. MR 99m:90108 Zbl 0973.90095

[Felipe 2009] R. Felipe, "Restrictive split and unital quasi-Jordan algebras", preprint 1-09-09, Centro de Investigación en Matemáticas, Guanajuato, 2009, Available at http://www.cimat.mx/reportes/ enlinea/I-09-09.pdf.

[Jordan et al. 1934] P. Jordan, J. von Neumann, and E. Wigner, "On an algebraic generalization of the quantum mechanical formalism”, Ann. of Math. 35:1 (1934), 29-64. MR 1503141 Zbl 0008.42103 
[Kolesnikov 2008] P. S. Kolesnikov, "Varieties of dialgebras, and conformal algebras", Sib. Math. J. 49:2 (2008), 257-272. MR 2009b:17002 Zbl 1164.17002

[Loday 1993] J.-L. Loday, "Une version non commutative des algèbres de Lie: les algèbres de Leibniz”, Enseign. Math. (2) 39:3-4 (1993), 269-293. MR 95a:19004 Zbl 0806.55009

[Loday 2001] J.-L. Loday, "Dialgebras", pp. 7-66 in Dialgebras and related operads, edited by J.L. Loday et al., Lecture Notes in Math. 1763, Springer, Berlin, 2001. MR 2002i:17004 Zbl 0999. 17002

[McCrimmon 1966] K. McCrimmon, "A general theory of Jordan rings", Proc. Nat. Acad. Sci. U.S.A. 56 (1966), 1072-1079. MR 34 \#2643 Zbl 0139.25502

[McCrimmon 1978] K. McCrimmon, "Jordan algebras and their applications", Bull. Amer. Math. Soc. 84:4 (1978), 612-627. MR 57 \#6115 Zbl 0421.17010

[McCrimmon 2004] K. McCrimmon, A taste of Jordan algebras, Springer, New York, 2004. MR 2004i:17001 Zbl 1044.17001

[Velásquez and Felipe 2008] R. Velásquez and R. Felipe, "Quasi-Jordan algebras", Comm. Algebra 36:4 (2008), 1580-1602. MR 2009b:17004 Zbl 1188.17021

[Velásquez and Felipe 2009] R. Velásquez and R. Felipe, "Split dialgebras, split quasi-Jordan algebras and regular elements”, J. Algebra Appl. 8:2 (2009), 191-218. MR 2010m:17024 Zbl 1188. 17022

[Zelmanov 1979] E. I. Zelmanov, "Jordan division algebras", Algebra i Logika 18:3 (1979), 286310. MR $81 \mathrm{~m}: 17021$

[Zelmanov 1983] E. I. Zelmanov, "Prime Jordan algebras, II", Sibirsk. Mat. Zh. 24:1 (1983), 89104. MR 85d:17011

Received February 19, 2010. Revised May 10, 2010.

RAÚL FELIPE

raulf@cimat.mx

CEnTRo de InVESTigación EN MATEMÁticas (CIMAT)

JALISCO S/N, COL. VALENCIANA

36240 Guanajuato, Gto.

MEXICO

and

Instituto de CibernéticA, MatemáticA y FísiCA (ICIMAF)

CALle F ESQUina A 15, No 309

VEDADO

10400 CiUdad de la Habana, Cuba 


\title{
PACIFIC JOURNAL OF MATHEMATICS
}

\author{
http://www.pjmath.org \\ Founded in 1951 by
}

E. F. Beckenbach (1906-1982) and F. Wolf (1904-1989)

\section{EDITORS}

V. S. Varadarajan (Managing Editor)

Department of Mathematics

University of California

Los Angeles, CA 90095-1555

pacific@math.ucla.edu

Vyjayanthi Chari

Department of Mathematics

University of California

Riverside, CA 92521-0135

chari@math.ucr.edu

\section{Robert Finn}

Department of Mathematics Stanford University

Stanford, CA 94305-2125

finn@math.stanford.edu

Kefeng Liu

Department of Mathematics

University of California

Los Angeles, CA 90095-1555

liu@math.ucla.edu
Darren Long

Department of Mathematics

University of California

Santa Barbara, CA 93106-3080

long@math.ucsb.edu

Jiang-Hua Lu

Department of Mathematics

The University of Hong Kong

Pokfulam Rd., Hong Kong jhlu@maths.hku.hk

Alexander Merkurjev

Department of Mathematics University of California

Los Angeles, CA 90095-1555 merkurev@math.ucla.edu
Sorin Popa

Department of Mathematics

University of California

Los Angeles, CA 90095-1555

popa@math.ucla.edu

Jie Qing

Department of Mathematics

University of California

Santa Cruz, CA 95064

qing@ cats.ucsc.edu

Jonathan Rogawski

Department of Mathematics

University of California

Los Angeles, CA 90095-1555

jonr@math.ucla.edu

\section{PRODUCTION}

pacific@math.berkeley.edu

Silvio Levy, Scientific Editor Matthew Cargo, Senior Production Editor

ACADEMIA SINICA, TAIPEI

CALIFORNIA INST. OF TECHNOLOGY

INST. DE MATEMÁTICA PURA E APLICADA

KEIO UNIVERSITY

MATH. SCIENCES RESEARCH INSTITUTE

NEW MEXICO STATE UNIV.

OREGON STATE UNIV.

\section{SUPPORTING INSTITUTIONS}

STANFORD UNIVERSITY
UNIV. OF BRITISH COLUMBIA
UNIV. OF CALIFORNIA, BERKELEY
UNIV. OF CALIFORNIA, DAVIS
UNIV. OF CALIFORNIA, LOS ANGELES
UNIV. OF CALIFORNIA, RIVERSIDE
UNIV. OF CALIFORNIA, SAN DIEGO
UNIV. OF CALIF., SANTA BARBARA

UNIV. OF CALIF., SANTA CRUZ

UNIV. OF MONTANA

UNIV. OF OREGON

UNIV. OF SOUTHERN CALIFORNIA

UNIV. OF UTAH

UNIV. OF WASHINGTON

WASHINGTON STATE UNIVERSITY

These supporting institutions contribute to the cost of publication of this Journal, but they are not owners or publishers and have no responsibility for its contents or policies.

See inside back cover or www.pjmath.org for submission instructions.

The subscription price for 2011 is US \$420/year for the electronic version, and \$485/year for print and electronic.

Subscriptions, requests for back issues from the last three years and changes of subscribers address should be sent to Pacific Journal of Mathematics, P.O. Box 4163, Berkeley, CA 94704-0163, U.S.A. Prior back issues are obtainable from Periodicals Service Company, 11 Main Street, Germantown, NY 12526-5635. The Pacific Journal of Mathematics is indexed by Mathematical Reviews, Zentralblatt MATH, PASCAL CNRS Index, Referativnyi Zhurnal, Current Mathematical Publications and the Science Citation Index.

The Pacific Journal of Mathematics (ISSN 0030-8730) at the University of California, c/o Department of Mathematics, 969 Evans Hall, Berkeley, CA 94720-3840, is published monthly except July and August. Periodical rate postage paid at Berkeley, CA 94704, and additional mailing offices. POSTMASTER: send address changes to Pacific Journal of Mathematics, P.O. Box 4163, Berkeley, CA 94704-0163.

PJM peer review and production are managed by EditFLOW ${ }^{\mathrm{TM}}$ from Mathematical Sciences Publishers.

PUBLISHED BY PACIFIC JOURNAL OF MATHEMATICS

at the University of California, Berkeley 94720-3840

A NON-PROFIT CORPORATION

Typeset in IATEX

Copyright $(2011$ by Pacific Journal of Mathematics 


\section{PACIFIC JOURNAL OF MATHEMATICS}

Volume $251 \quad$ No. $1 \quad$ May 2011

An analogue of the Cartan decomposition for $p$-adic symmetric spaces of split $\quad 1$ $p$-adic reductive groups

PATRICK DELORME and VINCENT SÉCHERRE

Unital quadratic quasi-Jordan algebras

RAÚL FELIPE

The Dirichlet problem for constant mean curvature graphs in $\mathbb{M} \times \mathbb{R}$ over unbounded domains

Abigail FolHa and Sofia Melo

Osgood-Hartogs-type properties of power series and smooth functions

BUMA L. FRIDMAN and DAOWEI MA

Twisted Cappell-Miller holomorphic and analytic torsions

RUNG-TZUNG HUANG

Generalizations of Agol's inequality and nonexistence of tight laminations

THILO KUESSNER

Chern numbers and the indices of some elliptic differential operators

PING LI

Blocks of the category of cuspidal $\mathfrak{s p}_{2 n}$-modules

VOLODYMYR MAZORCHUK and CATHARINA STROPPEL

A constant mean curvature annulus tangent to two identical spheres is Delauney

SUNG-HO PARK

A note on the topology of the complements of fiber-type line arrangements in $\mathbb{C P} \mathbb{P}^{2}$

Sheng-Li Tan, Stephen S.-T. YaU and Fei Ye

Inequalities for the Navier and Dirichlet eigenvalues of elliptic operators

QIAOLING WANG and CHANGYU XIA

A Beurling-Hörmander theorem associated with the Riemann-Liouville operator

XUECHENG WANG 